07

\title{
Влияние высоты барьера затвор-канал на характеристики детектирования полевого транзистора в сверхвысокочастотном и терагерцовом диапазонах
}

\author{
(C) С.А. Королев, ${ }^{1}$ Н.В. Востоков, ${ }^{1,2}$ Н.В. Дьяконова, ${ }^{3}$ В.И. Шашкин ${ }^{1,2}$ \\ ${ }^{1}$ Институт фризики микроструктур РАН, \\ 603950 Нижний Новгород, Россия \\ ${ }^{2}$ Нижегородский государственный университет им. Н.И. Лобачевского, \\ 603950 Нижний Новгород, Россия \\ ${ }^{3}$ Université Montpellier 2, \\ 34095 Montpellier, France \\ e-mail: pesh@ipmras.ru
}

(Поступило в Редакцию 28 марта 2016 г. В окончательной редакции 3 октября 2016 г.)

Теоретически исследованы детектирующие свойства полевого транзистора с пониженной высотой барьера Шоттки затвора. Рассмотрены различные схемы детектора. Найдены распределения напряжения и тока вдоль канала, входной импеданс транзистора, чувствительность и мощность эквивалентная шуму (NEP). Проанализировано влияние высоты барьера Шоттки затвора на перечисленные характеристики.

DOI: 10.21883/JTF.2017.05.44449.1821

\section{Введение}

В качестве чувствительного элемента неохлаждаемых детекторов сверхвысокочастотного (СВЧ) и терагерцового (ТГц) диапазонов используются ячейка Голея, пироэлектрический элемент, болометр, диод с барьером Шоттки, полевой транзистор и др. [1] Последнее двадцатилетие ознаменовалось успехами в создании детекторов на основе полевых транзисторов, способных принимать сигнал в ТГц диапазоне [2-9]. Первый такой детектор был создан на базе транзистора с высокой подвижностью электронов (high electron mobility transistor, HEMT) и принимал сигнал на частоте $2.5 \mathrm{THz}$ [2]. Вслед за исследованиями транзисторов на основе соединений III и V групп (GaAs/AlGaAs, GaInAs/AlGaAs, GaN/GaAlGaN и др.) [2-5] была продемонстрирована возможность детектирования на кремниевом транзисторе [6,7]. Хорошо развитая кремниевая технология позволяет интегрировать большое число транзисторов на единой пластине, делая возможным создание матричных приемников $[8,9]$.

Теоретическое описание процесса детектирования полевого транзистора в СВЧ и ТГц диапазонах можно найти в работах [10-15]. На низких частотах, когда емкостной ток через переход затвор-канал пренебрежимо мал, можно пользоваться статическими уравнениями транзистора [10]. В ТГц диапазоне емкостной ток, как правило, сравним с током в канале, и его нужно учитывать. В этом случае удобно представлять транзистор в виде двухпроводной линии электропередачи, где одним проводником является затвор, а вторым - канал [13]. На частотах выше частоты релаксации импульса свободных носителей заряда возможно резонансное детектирование, при этом движение носителей заряда в канале описывается уравнением Эйлера [11,12].
Как правило, в полевых транзисторах ток проводимости через переход затвор-канал пренебрежимо мал. В случае контакта металл-полупроводник это эквивалентно большой высоте барьера Шоттки $(\Delta \sim 1 \mathrm{eV})$. $\mathrm{B}$ настоящей работе исследуются линейные и детектирующие свойства полевого транзистора с пониженной высотой барьера $(\Delta \sim 0.1-0.3 \mathrm{eV})$. Снижение высоты барьера приводит к тому, что активная составляющая проводимости перехода затвор-канал становится сравнимой с емкостной составляющей, а также с проводимостью канала, что вызывает качественные изменения свойств транзистора. Снижение высоты барьера возможно разными способами. Например, за счет выращивания на границе металл-полупроводник тонкого слоя кремния [16] или за счет изотипного $\delta$-легирования полупроводника вблизи границы с металлом [17].

\section{Модель линии передачи для транзистора}

Структуру и электрическую схему полевого транзистора с пониженной высотой барьера иллюстрирует рис. 1. Закон изменения тока в канале $I$ и напряжения перехода затвор-канал $V$ от координаты $x$ и времени $t$ описывается системой уравнений

$$
\left\{\begin{array}{l}
l \frac{\partial V}{\partial x}=-\rho_{\mathrm{ch}}(V) I \\
-\frac{1}{l} \frac{\partial I}{\partial x}=j_{j}(V)+\frac{\partial}{\partial t}\left[c_{j}(V) V\right] .
\end{array}\right.
$$

Здесь $\rho_{\text {ch }}$ слоевое сопротивление канала, $j_{j}-$ плотность тока проводимости через переход затвор-канал, $c_{j}$ - удельная емкость перехода затвор-канал, $l-$ 


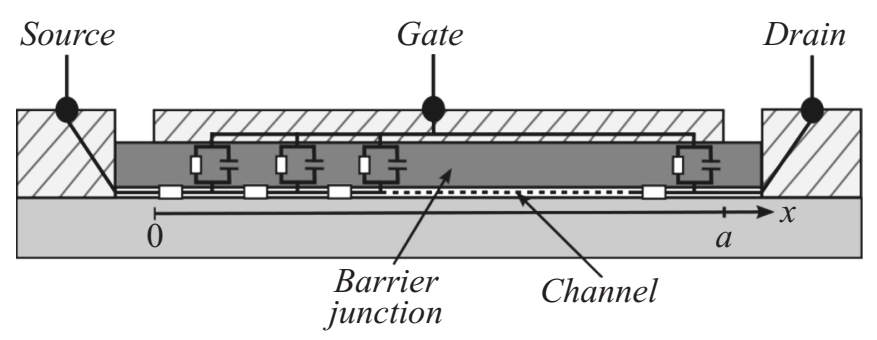

Рис. 1. Структура и электрическая схема полевого транзистора с переходом затвор-канал в виде контакта Шоттки с произвольной высотой барьера.

ширина затвора. В настоящей работе изучается детектирование сигнала малой амплитуды $\left(|V| \ll k_{\mathrm{B}} T\right.$, где $k_{\mathrm{B}}$ - постоянная Больцмана, $T$ - температура), поэтому эффекты, связанные с зависимостью $\Delta(V)[18,19]$, оказываются несущественными, и $\Delta$ полагается заданной постоянной величиной. Воспользуемся выражением для вольт-амперной характеристики контакта Шоттки [19]:

$$
j_{j}(V)=j_{S T}\left[\exp \left(\frac{V}{n V_{T}}\right)-1\right],
$$

где $\quad j_{S T}=n V_{T} / \rho_{j}, \quad V_{T}=k_{\mathrm{B}} T / e, \quad \rho_{j}=n V_{T} /\left(A^{* *} T^{2}\right) \times$ $\times \exp \left[\Delta /\left(k_{\mathrm{B}} T\right)\right]-$ удельное дифференциальное сопротивление контакта Шоттки при $V=0, A^{* *}$ - эффективная постоянная Ричардсона, $n-$ фактор неидеальности, $e$ - элементарный заряд. Поверхностная концентрация электронов в канале дается выражением [12]

$$
N(V)=\frac{C \eta V_{T}}{e} \ln \left[1+\exp \left(\frac{V-V_{\mathrm{thr}}}{\eta V_{T}}\right)\right],
$$

где $C=\varepsilon_{0} \varepsilon_{s} / d-$ геометрическая удельная емкость перехода затвор-канал, $\varepsilon_{s}$ - диэлектрическая проницаемость полупроводника, $\varepsilon_{0}$ - электрическая постоянная, $d$ - глубина залегания канала, $V_{\mathrm{thr}}$ - пороговое напряжение. Вблизи порогового напряжения $\left(\left|V-V_{\mathrm{thr}}\right| \lesssim V_{T}\right)$ формула (3) является приближенной с подгоночным параметром $\eta$. Удельная емкость перехода находится дифференцированием (3) по $V$

$$
c_{j}(V)=e \frac{d N}{d V}=C\left[1+\exp \left(-\frac{V-V_{\mathrm{thr}}}{\eta V_{T}}\right)\right]^{-1} .
$$

Поверхностное сопротивление канала $\rho_{\text {ch }}$ выражается через $N$ известным образом:

$$
\rho_{\mathrm{ch}}(V)=\frac{1}{e N(V) \mu},
$$

где $\mu-$ подвижность электронов в канале. Далее при расчетах и построении графиков будем использовать значения параметров, характерные для транзисторов на основе GaAs: $\varepsilon_{s}=13, A^{* *}=10 \mathrm{~A} \cdot \mathrm{cm}^{-2} \mathrm{~K}^{-2}$ [15], $n=1.1, \mu=1000 \mathrm{~cm}^{2} \mathrm{~V}^{-1} \mathrm{~s}^{-1}, \eta=1.9[5], a=100 \mathrm{~nm}$, $l=10 \mu \mathrm{m}, d=30 \mathrm{~nm}, V_{\mathrm{thr}}=-0.3 \mathrm{~V}, u=10 \mathrm{mV}$. Температура $T=300 \mathrm{~K}$.
В настоящей работе рассматривается режим работы детектора без смещения. Подробное исследование детектирующих свойств транзистора от напряжения смещения выполнено, например в [5], где было, в частности, показано негативное влияние тока утечки затвора на величину отклика. В случае малой амплитуды сигнала на выводах транзистора $u \ll V_{T}$ напряжение на переходе затвор-канал $V$ можно искать в виде разложения

$$
V \approx V_{1}+V_{2}
$$

где $V_{1}, V_{2}$ - некоторые функции координаты $x$ и времени $t$, которые пропорциональны соответственно первой и второй степеням $u$. Линейные характеристики транзистора определяются слагаемым $V_{1}$; за детектирование отвечает слагаемое $V_{2}$.

Пусть на выводы транзистора поступает гармонический сигнал с круговой частотой $\omega$. Решая (1) в линейном приближении, мы находим

$$
\begin{gathered}
V_{1}=u \operatorname{Re}(X(x) \exp (i \omega t)), \\
X(x)=C_{1} \cosh (\gamma x)+C_{2} \sinh (\gamma x),
\end{gathered}
$$

где $\gamma=\left(1+i \omega \rho_{j} c_{j 0}\right)^{1 / 2}, c_{j 0}=C /\left(1+\exp \left[V_{\mathrm{thr}} /\left(\eta V_{N}\right)\right]\right)-$ удельная емкость перехода затвор-канал при $V=$ $=0$, константы $C_{1}, C_{2}$ определяются граничными условиями. Здесь и далее черта над переменными означает, что они имеют безразмерный вид. Единица безразмерной длины $-\lambda_{0}=\left(\rho_{j} / \rho_{\mathrm{ch} 0}\right)^{1 / 2}$, где $\rho_{\mathrm{ch} 0}=\left[\mu C \eta V_{N} \ln (1+\right.$ $\left.\left.+\exp \left[-V_{\mathrm{thr}} /\left(\eta V_{N}\right)\right]\right)\right]^{-1}$ - поверхностное сопротивление канала при $V=0$. Как видно из (7), сигнал спадает на масштабе длины $\lambda=\lambda_{0} /|\gamma|=\left|z_{j} / \rho_{\text {ch } 0}\right|^{1 / 2}=\lambda_{0}[1+$ $\left.+\left(f / f_{0}\right)^{2}\right]^{-1 / 4}$. Здесь $z_{j}=\rho_{j} /\left(1+i \omega \rho_{j} c_{j 0}\right)-$ удельный импеданс перехода затвор-канал, $f=\omega /(2 \pi), f_{0}=$ $=\left(2 \pi \rho_{j} c_{j 0}\right)^{-1}-$ характерная частота перехода затвор-канал, при которой $\operatorname{Re} z_{j}=-\operatorname{Im} z_{j}$. Зависимость $\lambda(f)$ для разных значений $\Delta$ приведена на рис. 2.

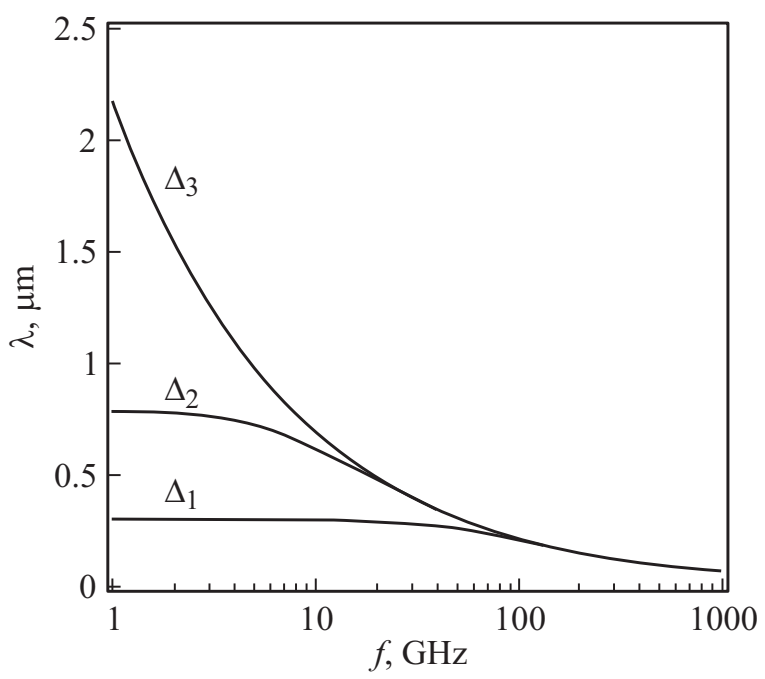

Рис. 2. Длина спадания сигнала от частоты: $\Delta_{1}=0.15 \mathrm{eV}$, $\Delta_{2}=0.2 \mathrm{eV}, \Delta_{3}=0.3 \mathrm{eV}$. 
На низких частотах $\left(f \ll f_{0}\right)$ длина спадания не зависит от частоты и равна $\lambda_{0}$. В этой области частот через переход затвор-канал течет в основном ток проводимости, величина которого определяется высотой барьера Шоттки. С увеличением высоты барьера длина спадания возрастает как $\lambda_{0} \propto \exp \left(\Delta /\left(2 k_{\mathrm{B}} T\right)\right)$. При $f \sim f_{0}$ включается емкостной канал протекания тока, импеданс перехода начинает уменьшаться, вследствие чего возрастает ток через переход, и сигнал в канале спадает быстрее ( $\lambda$ уменьшается). На больших частотах $\left(f \gg f_{0}\right)$ длина спадания не зависит от высоты барьера и равна $\lambda=\left(2 \pi f \rho_{\mathrm{ch} 0} c_{j 0}\right)^{-1 / 2}$. Если длина затвора $a \ll \lambda$ (короткий затвор), то напряжение на переходе $V_{1}$ практически не зависит от $x$, и транзистор можно рассматривать как элемент с сосредоточенными параметрами. В противном случае, когда $a \gtrsim \lambda$ (длинный затвор), нужно пользоваться моделью с распределенными параметрами.

Чтобы исследовать детектирующие свойства транзистора, мы должны учесть второе слагаемое в разложении (6). Решая (1) в этом приближении, мы найдем постоянное напряжение на переходе

$V_{2}=\frac{u^{2}}{4} \int_{0}^{\bar{x}} \Omega(\xi) \sinh (x-\xi) d \xi+D_{1} \cosh (\bar{x})+D_{2} \sinh (\bar{x})$,

где $\Omega(\xi)=|X(\xi)|^{2} /\left(n V_{N}\right)-d^{2} / d \xi^{2}\left[|X(\xi)|^{2}\right] /\left(\eta V_{N}\right), \quad$ константы $D_{1}, D_{2}$ определяются граничными условиями. Первое слагаемое $\Omega(\xi)$ отвечает детектированию на барьере затвор-канал, второе слагаемое - детектированию в канале. $V_{T}$ - характерное напряжение, на котором проявляются нелинейные свойства барьера,

$$
V_{N}=V_{T}\left[1+\exp \left(\frac{V_{\mathrm{thr}}}{\eta V_{T}}\right)\right] \ln \left[1+\exp \left(-\frac{V_{\mathrm{thr}}}{\eta V_{T}}\right)\right]
$$

- характерное напряжение, на котором проявляются нелинейные свойства канала. Значение $V_{N}$ зависит от величины порогового напряжения $V_{\text {thr }}$. В предельных случаях при отрицательном пороговом напряжении $\left(V_{\mathrm{thr}}<0,\left|V_{\mathrm{thr}}\right| \gg \eta V_{T}\right) V_{N}=\left|V_{\mathrm{thr}}\right| / \eta$, если пороговое напряжение положительно $\left(V_{\mathrm{thr}}>0,\left|V_{\mathrm{thr}}\right| \gg \eta V_{T}\right)$, то $V_{N}$ достигает минимального значения $V_{N}=V_{T}$.

\section{Схемы детектора и соответствующие распределения напряжения и тока в транзисторе}

Без использования дополнительных элементов существует 24 неэквивалентных способа включения транзистора в схему детектора: 4 варианта включения в высокочастотную (ВЧ) цепь и 6 вариантов включения в низкочастотную (НЧ) цепь (рис. 3). Способ включения будем обозначать парой натуральных чисел $\{m, n\}$, где $m$ - номер способа включения в ВЧ цепь, а $n$ - номер способа включения в НЧ цепь в соответствии с рис. 3.
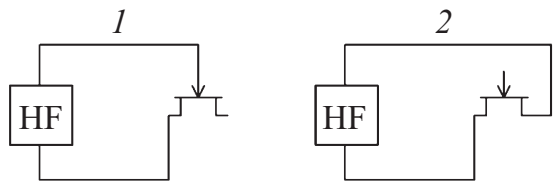

3
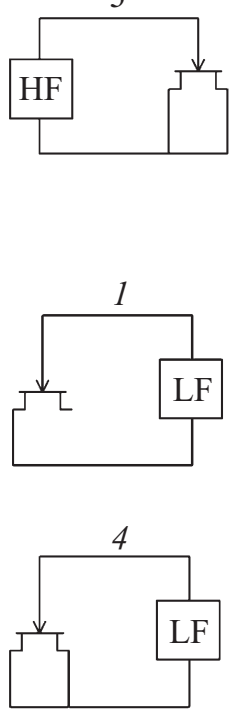
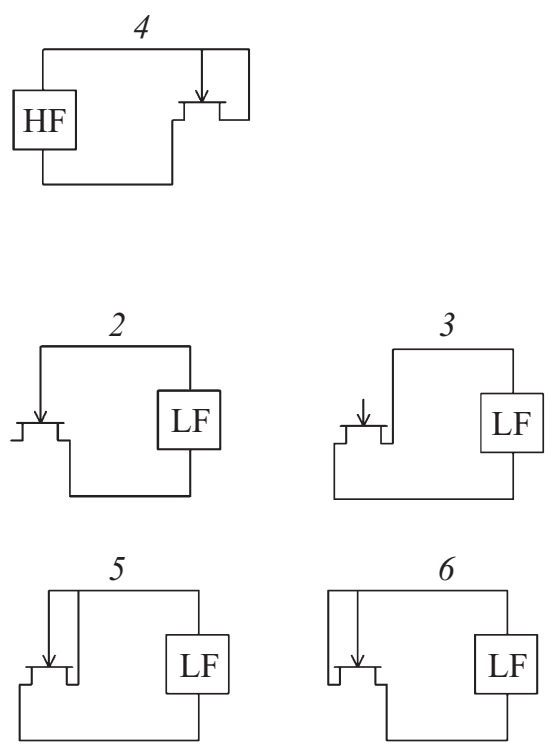

Рис. 3. Способы включения полевого транзистора в ВЧ и НЧ цепи детектора.

ВЧ цепь содержит антенну, а НЧ цепь - нагрузку с сопротивлением $R_{l}$. Способ включения определяет граничные условия для системы (1).

Для примера рассмотрим способ включения $\{1,5\}$. В этом случае граничные условия имеют вид

$$
\begin{gathered}
\left.V\right|_{x=0}=u,\left.\quad I\right|_{x=a}=0, \quad \text { для ВЧ сигнала, } \\
\left.V\right|_{x=0}=-V_{l},\left.\quad V\right|_{x=a}=0, \quad \text { для НЧ сигнала, }
\end{gathered}
$$

где $V_{1}$ - падение напряжения на сопротивлении нагрузки. Для того чтобы найти $V_{1}$, нужно принять во внимание дополнительное соотношение $I_{1}\left(V_{1}\right) R_{1}=V_{1}$, где $I_{1}-$ ток через сопротивление нагрузки. Учитывая (10a), (10b) из (7) и (8) получаем

$$
\begin{aligned}
V_{1}= & u \operatorname{Re}(X(x) \exp (i \omega t)), \quad X(x)=\frac{\cosh [\gamma(\bar{a}-\bar{x})]}{\cosh [\gamma \bar{a}]}, \\
V_{2}= & \frac{u^{2}}{4} \int_{0}^{\bar{x}} \Omega(\xi) \sinh (\bar{x}-\xi) d \xi-\frac{u^{2}}{4} \int_{0}^{\bar{a}} \Omega(\xi) \\
& \times \frac{\sinh (\bar{a}-\xi) \sinh (\bar{x})}{\sinh (\bar{a})} d \xi-V_{l} \frac{\sinh (\bar{a}-\bar{x})}{\sinh (\bar{a})} .
\end{aligned}
$$

Ток в канале, который также можно представить в виде двух слагаемых $I \approx I_{1}+I_{2}$, находится из первого урав- 


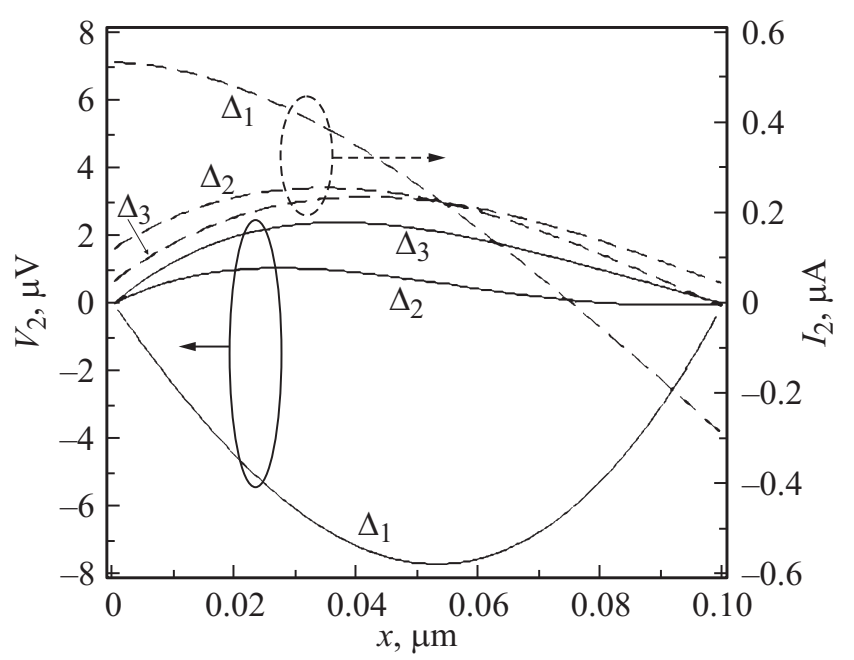

Рис. 4. Постоянные составляющие напряжения на переходе затвор-канал и тока в канале от координаты вдоль канала для способа включения $\{1,5\}: \Delta_{1}=0.15 \mathrm{eV}, \Delta_{2}=0.2 \mathrm{eV}$, $\Delta_{3}=0.3 \mathrm{eV}, f=300 \mathrm{GHz}$.

нения системы (1) подстановкой (6) с учетом (11) и (12):

$$
\begin{gathered}
I_{1}=\frac{l u}{\sqrt{\rho_{\operatorname{ch} 0 \rho_{j}}}} \operatorname{Re}\left(-\frac{d X}{d \bar{x}} \exp (i \omega t)\right), \\
-\frac{d X}{d \bar{x}}=\gamma \frac{\sinh [\gamma(\bar{a}-\bar{x})]}{\cosh [\gamma \bar{a}]}, \\
I_{2}=\frac{l}{\sqrt{\rho_{\operatorname{ch} 0} \rho_{j}}}\left\{-\frac{u^{2}}{4} \int_{0}^{\bar{x}} \Omega(\xi) \cosh (\bar{x}-\xi) d \xi\right. \\
+\frac{u^{2}}{4} \int_{0}^{\bar{a}} \Omega(\xi) \frac{\sinh (\bar{a}-\xi) \cosh (\bar{x})}{\sinh (\bar{a})} d \xi \\
\left.+\frac{u^{2}}{2 V_{N}} \operatorname{Re}\left(-X \frac{d X}{d \bar{x}}\right)-V_{l} \frac{\cosh (\bar{a}-\bar{x})}{\sinh (\bar{a})}\right\} .
\end{gathered}
$$

На рис. 4 построены зависимости постоянных составляющих напряжения и тока $V_{2}(x)$ и $I_{2}(x)$ для разных значений $\Delta$ в случае, когда сопротивление нагрузки равно нулю. При $\Delta=\Delta_{3}=0.3 \mathrm{eV}$ детектирование сигнала осуществляется в основном в канале. При этом происходит накопление электронов в канале, что приводит к возникновению положительного напряжения на переходе. При снижении высоты барьера электроны начинают эффективно удаляться из канала через барьер, что ведет к появлению отрицательного постоянного напряжения. Зависимость $I_{2}(x)$ также качественно меняет вид при снижении высоты барьера. При детектировании сигнала только в канале $\left(\Delta=\Delta_{3}\right)$ постоянный ток направлен в одну сторону во всей области канала. Выделенное направление характеризуется меньшим сопротивлением (большей концентрацией электронов) при протекании ВЧ тока в канале. При снижении высоты барьера возникает постоянная составляющая тока через барьер, которая в канале направлена как к истоку, так и к стоку.

\section{Входной импеданс транзистора}

Входной импеданс транзистора зависит от способа включения в ВЧ цепь детектора:

$$
\begin{gathered}
Z=\frac{\sqrt{\rho_{\operatorname{ch} 0 z_{j}}}}{l} \operatorname{coth}(\gamma \bar{a}), \quad m=1, \\
Z=\frac{2 \sqrt{\rho_{\operatorname{ch} 0 z_{j}}} \tanh \left(\frac{\gamma \bar{a}}{2}\right), \quad m=2,}{l} \ln \left(\frac{2 \sqrt{\rho_{\operatorname{ch} 0 z_{j}}}}{2 l} \operatorname{coth}\left(\frac{\gamma \bar{a}}{2}\right), \quad m=3,\right. \\
Z=\frac{\sqrt{\rho_{\operatorname{ch} 0 z_{j}}}}{l} \tanh (\gamma \bar{a}), \quad m=4 .
\end{gathered}
$$

На рис. 5, а построена зависимость $\operatorname{Re}(Z(f)), \operatorname{Im}(Z(f))$ для $f=0-\infty \mathrm{Hz}$. Из нее видно, что схемы включения транзистора в ВЧ цепь детектора можно разбить на две группы: $m=1,3$ и $m=2,4$. Отличия между этими группами проявляются, когда затвор транзистора короткий $(a \ll \lambda)$. В этом случае для подключений $m=1,3$ входной импеданс равен импедансу перехода затвор-канал
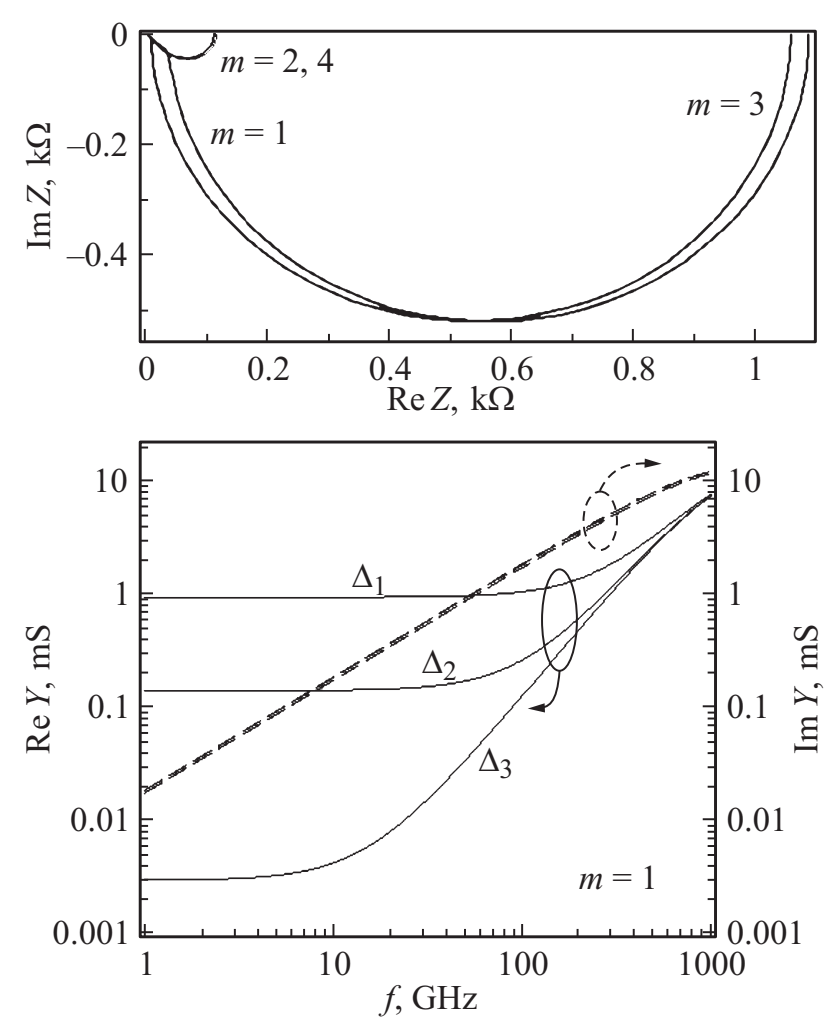

Рис. 5. $a-$ входной импеданс транзистора от частоты при $\Delta=0.15 \mathrm{eV} ; b-$ входной адмиттанс транзистора от частоты (сплошная кривая - действительная часть, штриховая кривая - мнимая часть) при $\Delta_{1}=0.15 \mathrm{eV}, \Delta_{2}=0.2 \mathrm{eV}$, $\Delta_{3}=0.3 \mathrm{eV}$. 
$Z_{j}=z_{j} /(a l)$, а для подключений $m=2,4-$ сопротивлению канала $R_{\operatorname{ch} 0}=\rho_{c h 0} \cdot a / l$. Если затвор транзистора длинный $(a \gg \lambda)$, то для всех способов подключения $Z=\left(R_{\mathrm{ch} 0} Z_{j}\right)^{1 / 2}$.

На рис. 5, $b$ построена зависимость обратной величины импеданса - адмиттанса $Y$ - от $f$ для способа включения $m=1$ при разных значениях $\Delta$. При $f \ll f_{0}$ мнимой частью адмиттанса транзистора можно пренебречь. В этом случае ток течет через активное сопротивление перехода затвор-канал $\left(Z_{j}=R_{j}=\right.$ $\left.=\rho_{j} /(a l), \gamma=1\right)$, адмиттанс транзистора является величиной вещественной и не зависящей от $f$. При $f \gg f_{0}$ ток через переход определяется емкостью $\left(Z_{j}=-i X_{j}=\right.$ $\left.=-i\left(\omega c_{j 0} a l\right)^{-1}, \gamma \bar{a}=\left(i \omega \rho_{\mathrm{ch} 0} c_{j 0}\right)^{1 / 2} a\right)$. Если при этом импеданс перехода остается по абсолютному значению больше сопротивления канала $\left(X_{j} \gg R_{\mathrm{ch} 0}\right)$, что эквивалентно условию короткого затвора $(a \ll \lambda)$, то адмиттанс транзистора преимущественно емкостной. Дальнейшее увеличение частоты приводит к возрастанию адмиттанса перехода. При достижении условия $X_{j} \ll R_{\text {ch } 0}$, что эквивалентно условию длинного затвора $(a \gg \lambda)$, действительная и мнимая части адмиттанса транзистора становятся равными: $\operatorname{Re}(Y)=\operatorname{Im}(Y)=\left[2 R_{\text {ch } 0} X_{j}\right]^{-1 / 2} \propto f^{1 / 2}$.

Мнимая часть входного адмиттанса транзистора практически не зависит от высоты барьера, так как определяется емкостным током через переход затвор-канал. При $f \ll f_{0}$ зависимость $\operatorname{Re}(Y)$ от $\Delta-$ экспоненциальная; при $f \gg f_{0}$ ток через переход определяется емкостью, поэтому зависимость $\operatorname{Re}(Y)$ от высоты барьера пропадает.

Согласование чувствительного элемента с антенной, имеющей импеданс $Z_{a}$, определяется коэффициентом передачи мощности

$$
\eta_{a}=\frac{4 \operatorname{Re}\left(Z_{a}\right) \operatorname{Re}(Z)}{\left|Z_{a}+Z\right|^{2}} .
$$

Антенна идеально согласована с чувствительным элементом, если $Z_{a}^{*}=Z$; в этом случае $\eta_{a}=1$. Для хорошего согласования транзистора с антенной в широкой полосе частот требуется разработка специальной антенны, импеданс которой будет зависеть от частоты требуемым образом. Возможно также использование подходящих согласующих устройств. В случае антенны с вещественным импедансом наилучшее согласование достигается, если входной импеданс транзистора тоже вещественный. Такая ситуация реализуется, во-первых, для транзистора с коротким затвором, подключенного к антенне по схемам $m=2,4$; во-вторых, для всех схем включения в диапазоне низких частот $f \ll f_{0}$, причем в этом диапазоне импеданс транзистора можно эффективно подстраивать изменением высоты барьера. Для транзистора с коротким затвором анализ согласования значительно упрощается, так как в этом случае транзистор может быть представлен простой эквивалентной схемой. Данное упрощение неприменимо к транзистору с длинным затвором, импеданс которого сложным образом зависит от частоты.

\section{Чувствительность транзистора}

Одной из основных характеристик детектирующего элемента является чувствительность: ампер-ваттная $S_{I}$ или вольт-ваттная $S_{V}=S_{I} R_{d}$, где $R_{d}-$ выходное дифференциальное сопротивление чувствительного элемента. Ампер-ваттная чувствительность определяется как отношение тока через сопротивление нагрузки $I_{l}$ к рассеиваемой на транзисторе мощности ВЧ сигнала $P$ при $R_{l}=0: S_{I}=I_{l} / P$. Для способа подключения к НЧ цепи $n=5$

$$
\begin{aligned}
& \left.I_{l}\right|_{R_{l}=0}=\left.I_{2}\right|_{x=0, V_{l}=0}=\frac{l u^{2}}{2 \sqrt{\rho_{\operatorname{ch} 0 \rho_{j}}}}\left\{\frac{1}{2} \int_{0}^{\bar{a}} \Omega(\xi)\right. \\
& \left.\quad \times \frac{\sinh (\bar{a}-\xi)}{\sinh (\bar{a})} d \xi+\left.\frac{1}{V_{N}} \operatorname{Re}\left(-X \frac{d X}{d \bar{x}}\right)\right|_{x=0}\right\} .
\end{aligned}
$$

Мощность $P=1 / 2 u^{2} \operatorname{Re}(1 / Z)$. Чувствительность транзистора с произвольной высотой барьера Шоттки затвоpa $S$ можно разложить на две части $\left(S_{N}\right.$ и $\left.S_{B}\right)$ : первая отвечает детектированию на нелинейности канала, вторая - на нелинейности барьера (под $S$ можно понимать как ампер-, так и вольт-ваттную чувствительность). Значения $S, S_{N}$ и $S_{B}$ во многом определяются способом включения транзистора в цепь детектора (таблица). Для способов $\{2,3\},\{3,3\}$ продетектированный как на одной, так и на другой нелинейности ток в НЧ цепи равен нулю из-за симметрии транзистора относительно истока и стока. Для способа $\{4,2\}$ продетектированные на разных нелинейностях сигналы вычитаются друг из друга, давая нулевую суммарную чувствительность. Есть подключения, когда после вычитания составляющих полная чувствительность остается отличной от нуля. Если продетектированные на разных нелинейностях сигналы складываются, то складываются и соответствующие чувствительности. Однако здесь можно выделить два качественно разных случая сложения (последние две строки таблицы). В первом случае максимального значения $S^{\max }$ чувствительность достигает в области параметров, где детектирование реализуется, в основном, на одной нелинейности; детектирование на другой подавлено. Такую ситуацию можно назвать конкуренцией нелинейностей. Во втором случае $S^{\max }$ достигается в области параметров, где своего максимального значения достигает каждая из составляющих $S_{N}$ и $S_{B}$.

Анализ показывает, что существует несколько способов подключения, дающих примерно одинаковые значения ампер-ваттной чувствительности $\sim e /\left(2 k_{\mathrm{B}} T\right)$. Среди них особый интерес представляет схема $\{1,5\}$, входящая в последнюю группу классификации (таблица). Подключения данной группы позволяют увеличить чувствительность транзистора за счет сложения продетектированных на разных нелинейностях сигналов. Максимальная ампер-ваттная чувствительность при подключении $\{1,5\}$ 
Значения чувствительности $S$ и ее составляющих $S_{N}$ и $S_{B}$ для различных способов включения транзистора в цепь детектора

\begin{tabular}{c|c|c|c}
\hline Способы включения & $S_{N}, S_{B}$ & $S$ & $S^{\max }$ \\
\hline$\{2,3\},\{3,3\}$ & $=0$ & $=0$ & $=0$ \\
$\{4,2\}$ & $>0$ & $=\left|S_{N}-S_{B}\right|=0$ & $=0$ \\
$\{1,2\},\{1,6\},\{4,6\}$ & $>0$ & $=\left|S_{N}-S_{B}\right| \neq 0$ & $=S_{N}-S_{B}$ \\
$\{1,1\},\{1,3\},\{1,4\},\{3,1\},\{3,2\}$, & $>0$ & & $\approx \max \left\{S_{N}-S_{B} \mid\right\}$ \\
$\{3,4\},\{3,5\},\{3,6\},\{4,3\},\{4,5\}$ & & $=S_{N}-S_{B}$ & $\left.=\max \left\{S_{N}\right\}, \max \left\{S_{B}\right\}\right\}$ \\
$\{1,5\},\{2,1\},\{2,2\},\{2,4\},\{2,5\}$, & $>0$ & & \\
$\{2,6\},\{4,1\},\{4,4\}$ & &
\end{tabular}

достигается в условиях короткого затвора $(a \ll \lambda)$

$$
S_{I}=\frac{1}{2} \frac{\frac{1}{2 n V_{T}}+\frac{1}{R_{j}}+\frac{1}{\eta V_{N}}\left[\frac{1}{R_{j}}+\frac{R_{\mathrm{ch} 0}}{6 X_{j}^{2}}\right]}{\frac{1}{R_{j}}+\frac{R_{\mathrm{ch} 0}}{3 X_{j}^{2}}}
$$

В предельном случае высокого барьера $\left(1 / R_{j}=0\right)$ детектирования на барьерной нелинейности нет, $S_{I}=$ $=1 /\left(4 \eta V_{N}\right)$. При отрицательном пороговом напряжении $\left(V_{\mathrm{thr}}<0,\left|V_{\mathrm{thr}}\right| \gg \eta V_{T}\right) S_{I}=1 /\left(4\left|V_{\mathrm{thr}}\right|\right)$, при положительном пороговом напряжении $\left(V_{\mathrm{thr}}>0,\left|V_{\mathrm{thr}}\right| \gg \eta V_{T}\right)$ $S_{I}=1 /\left(4 \eta V_{T}\right)$, что совпадает соответственно с формулами (17) и (24) из [14]. Если же барьер достаточно низкий $\left(1 / R_{j} \gg R_{\mathrm{ch} 0} /\left(3 X_{j}^{2}\right)\right)$, тогда $(18)$ достигает наибольшего значения

$$
S_{I}^{\max }=\frac{1}{4 n V_{T}}+\frac{1}{2 \eta V_{N}} .
$$

Согласно (9), $\min \left\{V_{N}\right\}=V_{T}$, поэтому максимальное значение ампер-ваттной чувствительности при подключении $\{1,5\} S_{I}^{\max }=1 /\left(2 V_{T}\right)(1 /(2 n)+1 / \eta)$. Вклад барьерной составляющей в (19) в 2 раза меньше чувствительности диода Шоттки, так как половина продетектированного на барьерной нелинейности тока течет в затвор, а не НЧ цепь. Составляющая чувствительности транзистора, связанная с детектированием в канале, в 2 раза больше соответствующей величины транзистора с высоким барьером, что объясняется синфазным изменением ВЧ напряжения и тока в первом случае (активная проводимость перехода) и противофазным изменением во втором (емкостная проводимость перехода). С увеличением длины затвора $a(a \gtrsim \lambda)$ ампер-ваттная чувствительность уменьшается для обеих составляющих. Для барьерной - это объясняется увеличением потерь мощности на сопротивлении канала; для канальной увеличением потерь мощности на сопротивлении канала и перехода. Здесь под потерянной мощностью подразумевается та часть мощности $P$, которая не дает вклада в продетектированный сигнал.

На рис. 6 приведена зависимость ампер-ваттной чувствительности $S_{I}$ от частоты входного сигнала $f$ для способа включения $\{1,5\}$ при различных значениях высоты барьера перехода. $S_{I}$ высокобарьерного транзистора $\left(\Delta=\Delta_{3}=0.7 \mathrm{~V}\right)$ не зависит от частоты сигнала в диапазоне до $\sim 1 \mathrm{THz}$, так как на этих частотах затвор

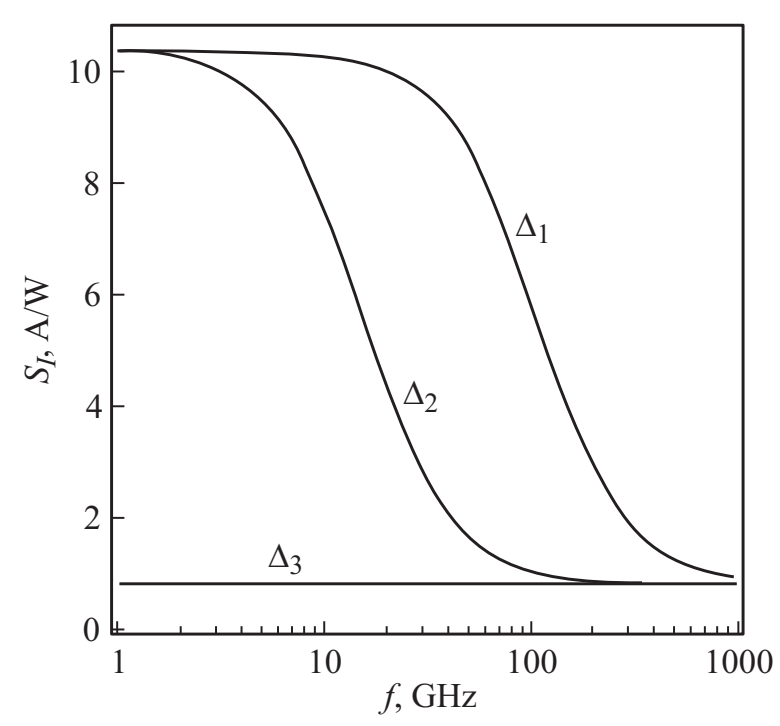

Рис. 6. Ампер-ваттная чувствительность транзистора от частоты для способа включения $\{1,5\}: \Delta_{1}=0.2 \mathrm{eV}, \Delta_{2}=0.3 \mathrm{eV}$, $\Delta_{3}=0.7 \mathrm{eV}$.

транзистора является коротким $(a \lesssim \lambda): S_{I}=1 /\left(4 \eta V_{N}\right)$. Снижение высоты барьера приводит к тому, что в диапазоне $f \ll f_{0}$ появляется добавка к ампер-ваттной чувствительности, равная $1 / 4\left[1 /\left(n V_{T}\right)+1 /\left(\eta V_{N}\right)\right]$, что дает в конечном счете (19).

\section{Мощность эквивалентная шуму детектора}

Характеристикой, учитывающей шумовые свойства детектора, является мощность эквивалентная шуму (noise equivalent power, NEP). При детектировании в режиме нулевого смещения основную роль играет тепловой шум с током через сопротивление нагрузки

$$
\left\langle\Delta I_{N}^{2}\right\rangle=\frac{4 k_{\mathrm{B}} T B_{\mathrm{LF}}}{R_{d}+R_{l}},
$$

где $B_{\mathrm{LF}}$ полоса НЧ цепи. Продетектированный ток выражается через мощность передаваемого транзистору 
антенной сигнала $P_{\text {in }}$ следующим образом:

$$
I_{l}=\frac{R_{d}}{R_{d}+R_{l}} S_{I} \eta_{a} P_{i n}
$$

Приравнивая $I_{l}$ и $\left\langle\Delta I_{N}^{2}\right\rangle^{1 / 2}$, полагая $B_{\mathrm{LF}}=1 \mathrm{~Hz}$, мы находим при идеальном согласовании по току в НЧ цепи $\left(R_{l} \ll R_{d}\right)$

$$
\mathrm{NEP}=\frac{1}{\eta_{a} S_{I}} \sqrt{\frac{4 k_{\mathrm{B}} T}{R_{d}}} .
$$

Ниже приведены выражения $R_{d}$ для всех способов подключения к НЧ цепи:

$$
\begin{array}{r}
R_{d}=\frac{\sqrt{\rho_{\operatorname{ch} 0 \rho_{j}}}}{l} \operatorname{coth}(\bar{a}), \quad n=1,2, \\
R_{d}=\frac{2 \sqrt{\rho_{\operatorname{ch} 0 \rho_{j}}}}{l} \tanh \left(\frac{\bar{a}}{2}\right), \quad n=3, \\
R_{d}=\frac{\sqrt{\rho_{\operatorname{ch} 0 \rho_{j}}}}{2 l} \operatorname{coth}\left(\frac{\bar{a}}{2}\right), \quad n=4, \\
R_{d}=\frac{\sqrt{\rho_{\operatorname{ch} 0 \rho_{j}}}}{l} \tanh (\bar{a}), \quad n=5,6 .
\end{array}
$$

Минимальное значение $\mathrm{NEP} \sim 10^{-12} \mathrm{~W} / \mathrm{Hz}^{1 / 2}$. На рис. 7 представлен график зависимости NEP от $f$ для способа включения $\{1,5\}$ при нескольких значениях $\Delta$. Снижение NEP с повышением частоты детектируемого сигнала связано с уменьшением входного импеданса транзистора, значение которого приближается к значению импеданса антенны. Лучшего согласования с антенной удается добиться при использовании транзистора с пониженной высотой барьера Шоттки затвора за счет уменьшения активного сопротивления перехода. Есть подключения, при которых NEP при понижении высоты барьера уменьшается не за счет улучшения согласования, а за счет увеличения

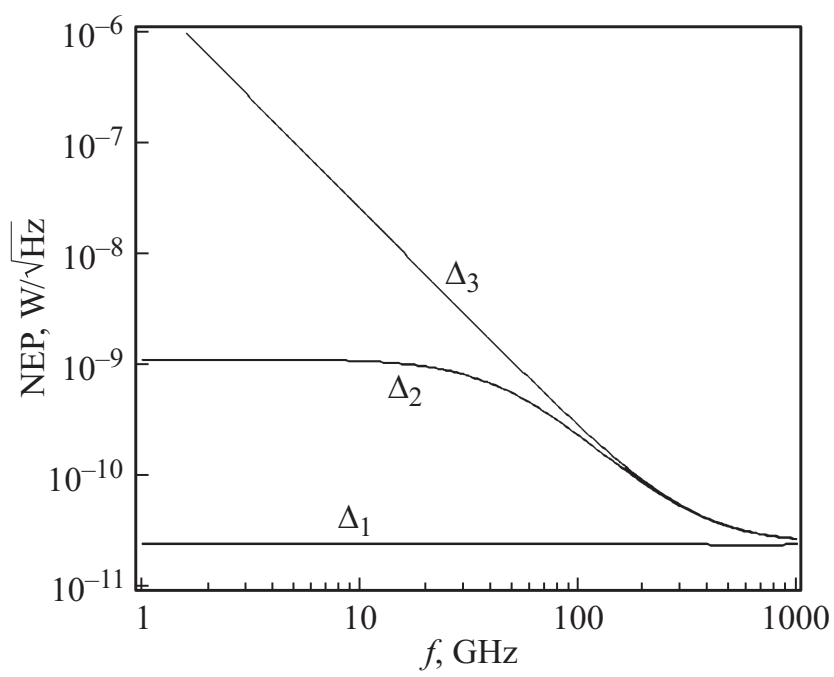

Pис. 7. Мощность эквивалентная шуму детектора от частоты для способа включения $\{1,5\}: \Delta_{1}=0.2 \mathrm{eV}, \Delta_{2}=0.3 \mathrm{eV}$, $\Delta_{3}=0.7 \mathrm{eV}, Z_{a}=100 \Omega$.

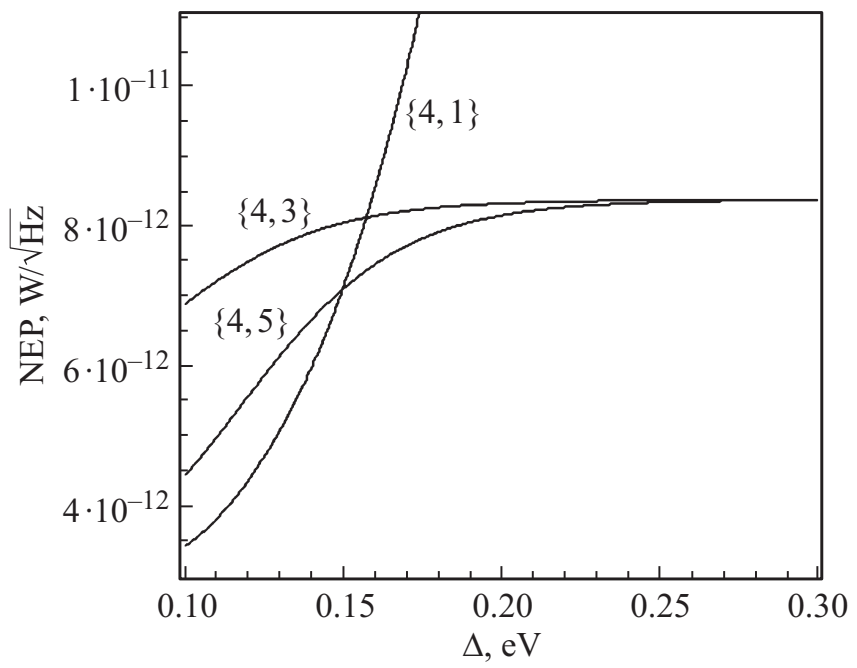

Рис. 8. Мощность эквивалентная шуму детектора от высоты барьера затвора для нескольких способов включения: $f=300 \mathrm{GHz}, Z_{a}=100 \Omega$.

чувствительности. Наиболее показательными здесь являются схемы $\{4,1\},\{4,3\}$ и $\{4,5\}$, для которых на рис. 8 изображена зависимость $\operatorname{NEP}(\Delta)$.

\section{Заключение}

В настоящей работе изучались линейные и детектирующие свойства полевого транзистора, в котором управление проводимостью канала осуществляется затвором с барьером Шоттки с пониженной высотой барьера. Для описания транзистора использовалась модель линии передачи, в которой одним из проводников является затвор, а другим - канал. Длина спадания сигнала в линии передачи дается выражением $\lambda=\lambda_{0}\left[1+\left(f / f_{0}\right)^{2}\right]^{-1 / 4}$, где $f_{0}$ - характерная частота перехода затвор-канал. При $f \ll f_{0}$ спадание определяется активной проводимостью перехода, при этом $\lambda=\lambda_{0}$. При $f \gg f_{0}$ спадание определяется емкостной проводимостью перехода, при этом $\lambda \propto f^{-1 / 2}$. Сравнение длины спадания $\lambda$ с длиной канала а позволяет сделать вывод о том, можно ли рассматривать транзистор как элемент с сосредоточенными параметрами $(a \ll \lambda)$ или нужно пользоваться моделью с распределенными параметрами $(a \gtrsim \lambda)$.

Граничные условия определяются способом включения транзистора в цепь детектора. Рассмотрены все возможные способы включения (всего 24 варианта). Найдено распределение ВЧ и постоянной составляющих напряжения и тока в канале транзистора.

Для всех способов включения найден входной импеданс транзистора, играющий определяющую роль при согласовании с антенной. При $f \ll f_{0}$ емкостной проводимостью перехода можно пренебречь, при этом импеданс транзистора становится чисто вещественным. Его можно изменять в определенных пределах варьированием высоты барьера Шоттки затвора, добиваясь лучшего 
согласования. В промежуточной области $f \sim f_{0}$ импеданс транзистора становится менее чувствительным к изменению высоты барьера в связи с шунтированием емкостью перехода. При $f \gg f_{0}$ активной проводимостью перехода можно пренебречь, зависимость импеданса транзистора от высоты барьера пропадает. В диапазоне $f \gtrsim f_{0}$ импеданс транзистора не может быть точно представлен эквивалентной схемой из конечного числа сосредоточенных элементов за исключением частного случая транзистора с коротким затвором $(a \ll \lambda)$. Для транзисторов с длинным затвором $(a \gg \lambda)$ на больших частотах $\left(f \gg f_{0}\right) \operatorname{Re} Z=-\operatorname{Im} Z \propto f^{-1 / 2}$.

Найдены ампер- и вольт-ваттная чувствительности транзистора. Снижение высоты барьера Шоттки затвора приводит к появлению дополнительной квадратичной нелинейности, связанной с протеканием тока проводимости через барьер. При некоторых способах включения транзистора в цепь детектора продетектированные на разных нелинейностях сигналы складываются. При оптимальном включении достигается максимальная ампер-ваттная чувствительность транзистора $S_{I}^{\max }=1 /\left(2 V_{T}\right)(1 /(2 n)+1 / \eta)$, которая на $1 /\left(4 n V_{T}\right)$ больше максимальной $S_{I}$ полевого транзистора с высоким барьером.

Рассчитана мощность эквивалентная шуму детектора на основе полевого транзистора с произвольной высотой барьера Шоттки затвора. Анализ различных схем включения показал, что снижение высоты барьера приводит к заметному уменьшению NEP детектора в диапазоне низких частот $\left(f \ll f_{0}\right)$, где за счет снижения активного сопротивления перехода значительно уменьшается входной импеданс транзистора. Для некоторых схем включения уменьшение NEP достигается за счет лучшей чувствительности транзистора с пониженной высотой барьера.

Работа выполнена при поддержке Программы РАН, проекта РФФИ № 16-52-76011 и Европейского проекта ERA.NET RUS Plus TERASENS.

\section{Список литературы}

[1] Sizov F., Rogalski A. // Progr. Quant. Electron. 2010. Vol. 34. N 5. P. $278-347$.

[2] Lü J.-Q., Shur M., Hesler J., Sun L., Weikle R. // IEEE Electr. Dev. Lett. 1998. Vol. 19. N 10. P. 373-375.

[3] Otsuji T., Hanabe M., Ogawara O. // Appl. Phys. Lett. 2004. Vol. 85. N 11. P. 2119-2121.

[4] Knap W., Deng Y., Rumyantsev S., Lü J.-Q., Shur M., Saylor C., Brunel L. // Appl, Phys. Lett. 2002. Vol. 80. N 18. P. 3433-3435.

[5] Knap W., Kachorovskii V., Deng Y., Rumyantsev S., Lü J.-Q., Gaska R., Shur M., Simin G., Hu X., Khan M., Saylor C., Brunel L. // J. Appl. Phys. 2002. Vol. 91. N 11. P. 9346-9353.

[6] Knap W., Teppe F., Meziani Y., Dyakonova N., Lusakowski J., Boeuf F., Skotnicki T., Maude D., Rumyantsev S., Shur M. // Appl. Phys. Lett. 2004. Vol. 85. N 4. P. 675-677.
[7] Tauk R., Teppe F., Boubanga S., Coquillat D., Knap W., Meziani Y., Gallon C., Boeuf F., Skotnicki T., FenouilletBeranger C., Maude D., Rumyantsev S., Shur M. // Appl. Phys. Lett. 2006. Vol. 89. N 25. P. 253511: 1-3.

[8] Öjefors E., Pfeiffer U., Lisauskas A., Roskos H. // IEEE J. Sol. St. Circ. 2009. Vol. 44. N 7. P. 1968-1976.

[9] Al Hadi R., Sherry H., Grzyb J., Zhao Y., Förster W., Keller H., Cathelin A., Kaiser A., Pfeiffer U. // IEEE J. Sol. St. Circ. 2012. Vol. 47. N 12. P. 2999-3012.

[10] Barrett R. // United States Patent. 1987. Pat. N 4.647.848.

[11] Dyakonov M., Shur M. // IEEE T. Electr. Dev. 1996. Vol. 43. N 3. P. $380-387$.

[12] Veksler D., Teppe F., Dmitriev A., Kachorovskii V., Knap W., Shur M. // Phys. Rev. B. 2006. Vol. 73. N 12. P. 125328: 1-10.

[13] Preu S., Kim S., Verma R., Burke P., Sherwin M., Gossard A. // J. Appl. Phys. 2012. Vol. 111. N 2. P. 024502: $1-9$.

[14] Kachorovskii V., Rumyantsev S., Knap W., Shur M. // Appl. Phys. Lett. 2013. Vol. 102. N 22. P. 223505: 1-4.

[15] Sakhno M., Golenkov A., Sizov F. // J. Appl. Phys. 2013. Vol. 114. N 16. P. 164503: 1-18.

[16] Costa J., Williamson F., Miller T., Beyzavi K., Nathan M., Mui D., Strite S., Moroç H. // Appl. Phys. Lett. 1991. Vol. 58. N 4. P. 382-384.

[17] Шашкин В.И., Мурель А.В., Дроздов Ю.Н., Данильцев В.М., Хрыкин О.И. // Микроэлектроника. 1997. Т. 26. № 1. C. 57-61.

[18] Шашкин В.И., Мурель А.В. // ФТП. 2004. Т. 38. Вып. 5. C. 574-579.

[19] Зи C.M. Физика полупроводниковых приборов. М.: Энергия, 1973. $656 \mathrm{c.}$ 